\title{
Conducta agresiva y ajuste personal y escolar en una muestra de estudiantes adolescentes españoles*
}

\author{
Aggressive Behavior and Personal and School Adjustment \\ in a Sample of Spanish Adolescent Students
}

Recibido: 8 de octubre de 2013 | Revisado: 14 de agosto de 2014 | Aceptado: 14 de agosto de 2014

\author{
ESTEFANÍA EsTÉVEZ LÓPEZ** \\ Universidad Miguel Hernández de Elche, España \\ TERESA ISABEL JiMÉNEZ GUTIÉRREZ*** \\ Universidad de Zaragoza, España
}

doi: 10.11144/Javeriana.upsy14-1.caap

Para citar este artículo: Estévez, E., \& Jiménez, T. I. (2015). Conducta agresiva y ajuste personal y escolar en una muestra de estudiantes adolescentes españoles. Universitas Psychologica, 14(1), 111-124. http://dx.doi.org/10.11144/Javeriana.upsy14-1.caap

* Este estudio ha sido financiado por el proyecto "La violencia escolar, de pareja y filio-parental en la adolescencia desde la perspectiva ecológica", con referencia PSI2012-33464 y subvencionado por el Ministerio de Economía y Competitividad de España.

** Profesora en el Área de Psicología Evolutiva y de la Educación en el Departamento de Psicología de la Salud en la Universidad Miguel Hernández de Elche. Investigadora del Equipo Lisis (www.uv.es/ lisis). Contacto principal para correspondencia editorial.Correo electrónico: eestevez@umh.es

**** Profesora en el Área de Psicología Evolutiva y de la Educación en el Departamento de Psicología y Sociología en la Universidad de Zaragoza. Campus de Teruel. Investigadora del Equipo Lisis (www. uv.es/lisis).Correo electrónico: tijimgut@unizar.es

\section{RESUMEN}

El objetivo del presente estudio fue determinar en qué medida la conducta agresiva hacia los iguales predice un mayor desajuste personal y escolar en los adolescentes agresores, chicos y chicas. Se utilizó una muestra de 1510 adolescentes españoles de Educación Secundaria Obligatoria. Los análisis de regresión logística mostraron que, en relación con el ajuste personal, el comportamiento agresivo predice significativamente y para ambos sexos altas puntuaciones en sintomatología depresiva, estrés percibido y soledad, y bajas puntuaciones en autoestima, satisfacción vital y empatía. En el ámbito escolar, la conducta agresiva se relacionó con bajas puntuaciones en implicación académica, afiliación con los compañeros, percepción de apoyo del profesor y actitud positiva hacia la escuela.

Palabras clave

adolescencia; conducta agresiva; ajuste personal; ajuste escolar; educación secundaria

\section{A B S T R A C T}

The aim of the present study was to determine to what extent aggressive behavior toward peers predicts problems of personal and school adjustment in aggressive adolescents, boys and girls. A sample of 1510 Spanish adolescents of compulsory secondary school was used. The logistic regression analyses showed that, in relation to personal adjustment, aggressive behavior significantly predicted for both sexes high scores in depressive symptoms, perceived stress and loneliness, and low scores on self-esteem, life satisfaction and empathy. For the school variables, aggressive behavior was associated with low scores on academic involvement, affiliation with peers, perceived teacher support and positive attitude toward school. Keywords

adolescence; aggressive behavior; personal adjustment; school adjustment; midschool education 
La literatura científica previa sobre la relación entre el comportamiento agresivo hacia los iguales en la escuela y variables de ajuste personal y escolar ha puesto de manifiesto en numerosas ocasiones el estrecho vínculo entre estos factores en población adolescente (Estévez, Herrero, Martínez, \& Musitu, 2006; Juvonen, Nishina, \& Graham, 2000). La mayor parte de estos estudios se ha centrado en analizar, por un lado, las consecuencias negativas de la agresión para las víctimas, como por ejemplo ansiedad, depresión o baja autoestima (Cava, Musitu, \& Murgui, 2007; Hunter, Mora-Merchán, \& Ortega, 2004; Sweeting, Young, West, \& Der, 2006) y, por otro, los factores de riesgo que explican el desarrollo de este tipo de comportamiento en los agresores.

Entre los factores de riesgo para el desarrollo de conductas agresivas hacia sus iguales identificados en adolescentes, se encuentran, entre otros: la baja autoestima (Martínez, Murgui, Musitu, \& Monreal, 2009; Wild, Flisher, Bhana, \& Lombard, 2004), la baja satisfacción vital y el alto grado de sentimiento de soledad (Estévez, Murgui, \& Musitu, 2008; Martínez, Povedano, Amador, \& Moreno, 2012), el elevado distrés psicológico (Villarreal-González, Sánchez-Sosa, Veiga, \& del Moral Arroyo, 2011), déficits en la habilidad empática (Evans, Heriot, \& Friedman, 2002; Mestre, Samper, Tur-Porcar, Richaud de Minci, \& Mesurado, 2012; Moreno, Estévez, Murgui, \& Musitu, 2009), la baja implicación académica (Álvarez-García et al., 2010; Gázquez \& PérezFuentes, 2010), la actitud negativa hacia los estudios, el centro y el profesorado (Estévez, Inglés, \& Martínez-Monteagudo, 2013; Molpeceres, Lucas, $\&$ Pons, 2000), y relaciones sociales negativas en la escuela con compañeros y profesores (Murray \& Murray, 2004; Reinke \& Herman, 2002).

La evidencia empírica es mucho más escasa, sin embargo, en relación con las consecuencias derivadas de la implicación en estas conductas agresivas para el ajuste personal y escolar del propio agresor. La conducta agresiva itiene consecuencias en la autoestima del agresor, su actitud hacia los estudios, su relación con los compañeros del aula?, por ejemplo. El presente estudio pretende contribuir al conoci- miento científico sobre esta cuestión, analizando la capacidad predictiva de la conducta agresiva sobre las principales variables de ajuste personal y escolar identificadas como relevantes en estudios previos y asociadas a este tipo de comportamiento en la escuela.

\section{Conducta agresiva en la escuela}

La conducta agresiva que muestran algunos adolescentes hacia sus iguales en el contexto escolar hace referencia a un conjunto de comportamientos dirigido hacia otros compañeros, con el objeto de causar un daño intencionado a nivel físico, verbal o relacional (Estévez, Jiménez, \& Musitu, 2008; Little, Henrich, Jones, \& Hawley, 2003). Estos comportamientos pueden obedecer a dos motivaciones: bien, responder de modo defensivo a una provocación previa, donde existe en numerosas ocasiones un sesgo en la interpretación de intenciones hostiles en el otro; bien, suponer una anticipación de beneficios controlada por refuerzos externos, como por ejemplo conseguir estatus social en el grupo o incluso la posición de líder en este (Emler, 2009; Little et al., 2003).

Desde un punto de vista epidemiológico, la agresión entre iguales es un hecho frecuente en los centros educativos y así ha sido constatado en numerosos estudios realizados con población escolar europea (Blaya, Debardieux, del Rey, \& Ortega, 2006; Olweus, 2005). Se ha considerado que la agresión entre compañeros es la forma más común de violencia escolar (Nansel et al., 2001), y si bien no hay acuerdo entre estudios respecto de los índices de prevalencia, sí que hay acuerdo en considerar la agresión entre compañeros como un obstáculo importante para el desarrollo del proceso de enseñanza-aprendizaje y el ajuste psicosocial y escolar, tanto de las víctimas como de los agresores (Astor, Pitner, Benbenishty, \& Meyer, 2002).

Como ya se ha señalado anteriormente, los antecedentes del comportamiento agresivo y los consecuentes de la agresión escolar para las víctimas han sido ampliamente analizados y puestos en evidencia en numerosos trabajos. Sin embargo, 
el estudio de las consecuencias de este comportamiento en los propios agresores ha quedado tradicionalmente soslayado en la investigación, mientras que es previsible que el desajuste mostrado a nivel conductual se relacione con problemas a otros niveles que pueden relegarlos a una situación cada vez más compleja y de difícil salida, si no se trata el problema convenientemente.

\section{Conducta agresiva y desajuste personal y escolar}

Los escasos trabajos en los que se ha considerado la conducta agresiva como variable predictora del desajuste en los adolescentes que la cometen, indican que estos se encuentran en un riesgo elevado de presentar dificultades emocionales, psicológicas y escolares a corto y largo plazo (Vanderbilt \& Augustyn, 2010). En el trabajo de revisión realizado por estos autores, se concluye que la implicación en conductas agresivas en la escuela se relaciona con posteriores problemas de salud y con distintos diagnósticos como cuadros de ansiedad y personalidad antisocial. Este y otros estudios también informan que participar en actos de agresión en la escuela incrementa las tasas de distrés psicológico, depresión, absentismo escolar y actitudes negativas hacia la escuela (Brunstein-Klomek et al., 2011; Zimmer-Gembeck \& Pronk, 2012).

En otras revisiones como la llevada a cabo por Ostrowsky (2010) se señala la importancia de seguir investigando en la relación existente entre la conducta agresiva y la autoestima de los agresores, siendo todavía inconcluyentes los resultados en la literatura científica actual, si bien el mayor consenso parece darse en la idea de que agresión y autoestima mantienen un vínculo bidireccional. Datos más asentados se encuentran sobre la agresión hacia los iguales en adolescentes como factor predictor de otras conductas antisociales posteriores y de conducta delictiva y violenta en la edad adulta (Olweus, 2011; Ttofi, Farrington, \& Lösel, 2012), un aspecto que refleja la espiral retroalimentada de desajuste psicosocial en la que se ven inmersos algunos adolescentes.

\section{El presente estudio}

La revisión de la investigación previa sobre las consecuencias del comportamiento agresivo en los adolescentes agresores presenta varias limitaciones. La investigación en población adolescente española es apenas existente. Además, los estudios no analizan indicadores de ajuste en varios dominios, sino que se circunscriben básicamente al ámbito individual (p. ej., autoestima) o comportamental (conducta antisocial o delictiva). El análisis conjunto de otros indicadores individuales (como el estrés, la soledad, la empatía o la satisfacción vital) y también escolares (como la afiliación con otros compañeros y la relación con el profesorado) podría aportar una imagen más completa de las dificultades asociadas a la situación de violencia escolar. Además, generalmente, los estudios no tienen en cuenta la variable sexo en sus análisis, mientras que la literatura científica señala la existencia de diferencias entre chicos y chicas en la implicación en conductas agresivas y variables relacionadas (EstebanGuitart, Rivas, \& Pérez, 2011; Postigo, González, Mateu, Ferrero, \& Martorell, 2009; Povedano, Estévez, Martínez, \& Monreal, 2012).

Teniendo en cuenta estas limitaciones, el presente estudio tiene como objetivo determinar, mediante análisis de regresión logística, en qué medida la conducta agresiva hacia los iguales predice un mayor desajuste psicosocial en los adolescentes agresores y, en particular, si esta conducta está asociada a altas o bajas puntuaciones en las siguientes variables individuales y escolares: autoestima, sintomatología depresiva, estrés percibido, satisfacción con la vida, sentimiento de soledad, empatía, actitud positiva hacia la escuela y el profesorado, implicación académica, afiliación con otros compañeros y apoyo percibido del profesor. Atendiendo a la literatura científica previa, se espera que la conducta agresiva sea un predictor estadísticamente significativo en ambos sexos para altas puntuaciones en sintomatología depresiva, estrés percibido y sentimiento de soledad y bajas puntuaciones en autoestima, satisfacción vital, empatía, actitud positiva hacia los estudios, implicación académica, afiliación con otros compañeros y apoyo percibido del profesor. 


\section{Método}

\section{Participantes}

Participaron en el estudio 1510 adolescentes (52\% chicos) escolarizados en nueve centros de enseñanza secundaria obligatoria en cursos de $1 .^{\circ}$ a 4. o de la Educación Secundaria Obligatoria (ESO). El rango de edad de los participantes fue de 12 a 16 años $(M$ $=13.4 ; \mathrm{DE}=1.25)$. La selección de los participantes se realizó a través de un muestreo aleatorio por conglomerados en las zonas geográficas de las comunidades valenciana y andaluza. Las unidades primarias del muestreo fueron las zonas geográficas rurales y urbanas de ambas comunidades. Las unidades secundarias fueron los institutos públicos y privados de cada zona, que se seleccionaron de forma aleatoria proporcional. Se llevaron a cabo análisis previos de diferencias de medias en función de la localización del centro (rural y urbano) y su condición de público o privado, en las variables objeto de estudio, sin identificarse diferencias estadísticamente significativas.

\section{Instrumentos}

Escala de Autoestima Global (Rosenberg Self-Esteem Scale [RSE]; Rosenberg, 1965; versión española de Echeburúa, 1995)

Esta escala consta de 10 ítems que aportan un índice general de autoestima, con una escala de respuesta tipo Likert de 4 puntos ( $1=$ muy en desacuerdo; 4 $=$ muy de acuerdo). Cinco ítems están formulados en sentido positivo (p. ej. "creo que tengo numerosas cualidades positivas") y cinco en sentido negativo ( $\mathrm{p}$. ej. "a veces me siento realmente inútil"). El coeficiente de consistencia interna medido a través del alpha de Cronbach para la presente muestra fue de 0.76 .

Escala de Depresión del Centro de Estudios Epidemiológicos (Center of Epidemiological Studies Depression Scale [CES-D]; Radloff, 1977; traducción bidireccional)

Esta escala se compone de 20 ítems que evalúan en una escala tipo Likert de 4 puntos ( $1=$ nunca; $4=$ siempre) sintomatología relacionada con el ánimo depresivo. El CESD proporciona un índice general de ánimo depresivo que no evalúa la depresión en sí misma, sino la sintomatología que habitualmente va asociada a ella (p. ej. "durante la última semana, me he sentido triste"). La fiabilidad del instrumento según el alpha de Cronbach obtenida en la presente muestra fue de 0.81 .

Escala de Estrés Percibido (Perceived Stress Scale [PSS]; Cohen, Kamarck, Mermelstein, 1983; versión breve española de cuatro items de Herrero $\mathbb{E}$ Meneses, 2006).

La PSS evalúa el grado en que el sujeto ha experimentado ciertas situaciones como estresantes en el último mes (p. ej. "en el último mes, he sentido que era incapaz de controlar las cosas más importantes de mi vida") mediante una escala tipo Likert con un rango de respuesta de 4 puntos $(1=$ nunca; 4 = siempre). El índice de consistencia interna (alpha de Cronbach) en la presente muestra fue de 0.64.

\section{Escala de Satisfacción con la Vida (Diener, Emmons, Larsen, $\mathcal{E}$ Griffin, 1985; versión española de Atienza, Pons, Balaguer, Eु García-Merita, 2000).}

Este instrumento se compone de 5 ítems que proporcionan un índice general de bienestar subjetivo percibido (p. ej., "no estoy contento con mi vida"). Los ítems se valoran mediante una escala tipo Likert de 4 puntos $(1=$ muy en desacuerdo; $4=$ muy de acuerdo). En el presente estudio, el índice de consistencia interna (alpha de Cronbach) fue de 0.74.

\section{Escala de Soledad UCLA ([UCLA Loneliness Scale]; Russell, Peplau, Ë Cutrona, 1980; versión española de Expósito $\mathbb{E}$ Moya, 1993).}

Esta escala consta de 20 ítems con opciones de respuesta tipo Likert de 4 puntos $(1=$ nunca; $4=$ siempre), que aportan una medida general de sentimiento de soledad (p. ej. , "con qué frecuencia te sientes aislado de los demás"). En la presente muestra el coeficiente de fiabilidad (alpha de Cronbach) fue de 0.89 . 
Escala de Empatía para Niños y Adolescentes

([Index of Empathy for Children and Adolescents, IECA]; Bryant, 1982; versión española de Mestre, Pérez-Delgado, Frías, Ė Samper, 1999).

Este instrumento aporta una evaluación general del nivel de empatía en niños y adolescentes (p. ej. , "me siento mal cuando veo que se hace daño a otra persona") a través de 22 ítems con una rango de respuesta tipo Likert de 4 puntos $(1=$ nunca; $4=$ siempre). En el presente estudio el alpha de Cronbach fue de 0.77 .

Escala de Actitud hacia la Autoridad Institucional (Reicher Eु Emler, 1985; traducción bidireccional).

Este escala se compone de 10 ítems que evalúan la actitud de los estudiantes hacia la escuela y el profesorado como institución y figuras de autoridad (p. ej. , "estoy de acuerdo con lo que hacen y dicen la mayoría de los profesores"). La escala de respuesta es tipo Likert de 4 puntos $(1=$ totalmente en desacuerdo; 4 = totalmente de acuerdo). En el presente estudio el alpha de Cronbach fue de 0.74 .

\section{Relaciones Interpersonales en la Escuela}

([Classroom Climate Scale, CES]; Moos

E3 Trickett 1973; versión española de

Fernández-Ballesteros $\mathcal{E}$ Sierra, 1989).

Se utilizó la dimensión de relaciones interpersonales en la escuela de la CES, que aporta una medida de la calidad del clima percibido en el aula respecto de las relaciones entre alumnos y entre alumnado y profesorado. Esta subescala se compone de 30 ítems con opciones de respuesta de verdadero o falso, que miden a su vez 3 dimensiones: Implicación Académica (p. ej. "a los alumnos les gusta ayudarse para hacer los deberes"), Afiliación entre Compañeros (p. ej. , "en esta clase se hacen muchas amistades" y Apoyo Percibido del Profesor (p. ej. , "los profesores muestran interés personal por sus alumnos"). El alpha de Cronbach obtenido en la presente muestra fue de 0.63 para Implicación Académica, 0.63 para Afiliación entre Compañeros y 0.68 para Apoyo Percibido del Profesor.
Escala de Conducta Agresiva (Little et al., 2003; traducción bidireccional).

Esta escala consta de 25 ítems con un rango de respuesta tipo Likert de 4 puntos $(1=$ nunca; $4=$ siempre) según la participación en comportamientos de carácter agresivo hacia iguales en población escolar. La escala mide dos tipos de conducta agresiva (manifiesta o directa, y relacional o indirecta) y tres funciones de la violencia (pura, reactiva e instrumental), dando lugar a seis dimensiones de la agresión: manifiesta pura (p. ej. , "soy una persona que se pelea con los demás”), manifiesta reactiva (p. ej. , "cuando alguien me hace daño o me hiere, le pego"), manifiesta instrumental (p. ej. , "amenazo a otros para conseguir lo que quiero"), relacional pura (p. ej., "soy una persona que chismorrea y cuenta rumores de los demás"), relacional reactiva (p. ej., "cuando alguien me enfada, le trato con indiferencia o dejo de hablarle") y relacional instrumental (p. ej., "para conseguir lo que quiero, desprecio a los demás"). El alpha de Cronbach de las seis dimensiones en el presente estudio osciló entre 0.72 y 0.87 .

\section{Procedimiento}

En primer lugar se contactó con la dirección de los centros educativos seleccionados. Tras confirmar telefónicamente su interés y participación voluntaria, se realizó un seminario informativo con la plantilla de profesorado para explicar los objetivos y alcance de la investigación, describir los instrumentos de evaluación, solicitar los permisos pertinentes y promover su participación. A continuación, se envió una carta explicativa de la investigación a los padres y madres de los alumnos mediante correo postal donde, además, se solicitó que expresaran por escrito su consentimiento en relación con la participación de sus hijos. Una vez recogido el consentimiento paterno, se acordaron con los centros las fechas para la aplicación de los instrumentos con los alumnos. La recogida de datos se llevó a cabo, con cada grupo, en su aula habitual y durante el horario regular del centro. En la aplicación participó un grupo de investigadores expertos y entrenados, que estuvo presente durante la admi- 
nistración de las escalas para proporcionar ayuda a los alumnos y supervisar la correcta cumplimentación de las mismas. El orden de administración de los instrumentos se contrabalanceó en cada aula y centro educativo. Se informó a los adolescentes que su participación en la investigación era voluntaria y anónima y que podían abandonar el estudio en cualquier momento del proceso.

\section{Resultados}

\section{Análisis estadísticos}

Con el fin de examinar y cuantificar la capacidad predictiva de la conducta agresiva sobre las variables de ajuste personal y escolar de interés, se calcularon análisis de regresión logística binaria siguiendo el procedimiento de regresión por pasos hacia delante basado en el estadístico de Wald. El análisis de regresión logística permite estimar la probabilidad de que ocurra un suceso o resultado (p. ej., alta sintomatología depresiva) en presencia de la variable predictora (p. ej., conducta agresiva). Esta probabilidad se estima mediante el estadístico Odd Ratio (OR). Un valor de la OR superior a 1, indica que el aumento de la variable independiente lleva asociado un incremento en la ventaja del suceso, es decir, en la probabilidad de que este ocurra. Por el contrario, un valor de la OR inferior a 1 , se interpreta como que el aumento de la variable independiente conlleva una disminución de la ventaja del suceso, es decir, una disminución en la probabilidad de ocurrencia del mismo (De Maris, 2003).

Para llevar a cabo estos análisis, las variables criterio (autoestima, sintomatología depresiva, estrés percibido, satisfacción con la vida, sentimiento de soledad, empatía, actitud positiva hacia la escuela y el profesorado, implicación académica, amistades en el aula y apoyo percibido del profesor) fueron dicotomizadas en función de los percentiles 25 y 75 para identificar la presencia alta o baja del constructo. La proporción de casos clasificados correctamente por los modelos logísticos osciló entre: 62.2\% (afiliación entre compañeros) y 85.1\% (estrés percibido) en la muestra de varones y $64.7 \%$ (afiliación entre compañeros) en la muestra de mujeres.
La conducta agresiva como variable predictora del ajuste personal y escolar en los chicos

En la muestra de varones, las $\mathrm{OR}$ indican que la probabilidad de presentar alta sintomatología depresiva aumenta 4.9 veces por cada punto que aumenta la puntuación en la escala de conducta agresiva. El estrés percibido aumenta 4.84 veces y el sentimiento de soledad 2.41 veces, conforme se incrementa la puntuación en conducta agresiva. Los resultados presentados en la Tabla 1 también indican que la probabilidad de presentar baja empatía, baja satisfacción vital y baja autoestima aumenta 6.7, 3.14 y 2.24 veces respectivamente, por cada punto que aumenta la conducta agresiva. Respecto de las variables de ajuste escolar, las OR indican que la probabilidad de presentar una baja actitud positiva hacia la escuela y el profesorado y una baja implicación en tareas escolares aumenta 2.91 y 1.31 veces, respectivamente, conforme aumenta la conducta agresiva. Un incremento semejante se observa para la baja afiliación y amistad con los compañeros de clase $(\mathrm{OR}=2.68)$ y el bajo apoyo del profesor $(\mathrm{OR}=3.90)$. En resumen y en el caso de los chicos, el orden de las variables según el valor de la OR de mayor a menor es el siguiente: bajo grado de empatía, alta sintomatología depresiva, alto estrés percibido, bajo apoyo del profesor, baja satisfacción vital, baja actitud positiva hacia la escuela y el profesorado, baja afiliación entre compañeros, alto sentimiento de soledad, baja autoestima y baja implicación académica.

\section{La conducta agresiva como}

variable predictora del ajuste

personal y escolar en las chicas

En la muestra de mujeres los resultados son muy similares a los encontrados en la de varones. Las OR que se presentan en la Tabla 2 indican que la probabilidad de exhibir alta sintomatología depresiva, estrés percibido y sentimiento de soledad aumenta $3.44,2.73$ y 2.35 veces, respectivamente, por cada punto que aumenta la puntuación en la escala de conducta agresiva. Para el resto de variables de 
TABLA 1

Regresión logística con conducta violenta como predictor en chicos

\begin{tabular}{lcccccccc}
\hline & & B & ET & Wald & p & OR & \multicolumn{2}{c}{ CI 95\% } \\
\hline Altas puntuaciones & & & & & & & Inferior & Superior \\
\hline Sintomatología depresiva & Conducta violenta & 1.59 & 0.24 & 42.07 & 0 & 4.9 & 3.03 & 7.92 \\
& Constante & -3.99 & 0.4 & 96.67 & 0 & 0.01 & & \\
Estrés percibido & Conducta violenta & 1.57 & 0.25 & 39.1 & 0 & 4.84 & 2.95 & 7.93 \\
& Constante & -4.15 & 0.42 & 96.91 & 0 & 0.02 & & \\
Sentimiento soledad & Conducta violenta & 0.88 & 0.21 & 16.36 & 0 & 2.41 & 1.57 & 3.69 \\
& Constante & -2.33 & 0.34 & 45.64 & 0 & 0.1 & & \\
\hline Bajas puntuaciones & & & & & & & & \\
\hline Autoestima & Conducta violenta & 0.81 & 0.21 & 14.33 & 0 & 2.24 & 1.47 & 3.42 \\
& Constante & -2.1 & 0.33 & 38.8 & 0 & 0.12 & & \\
Satisfacción vital & Conducta violenta & 1.14 & 0.21 & 27.62 & 0 & 3.14 & 2.05 & 4.82 \\
Grado de empatía & Constante & -2.56 & 0.34 & 55.13 & 0 & 0.07 & & \\
& Conducta violenta & 1.90 & 0.24 & 62.99 & 0 & 6.7 & 4.19 & 1.72 \\
Actitud hacia escuela/profesorado & Constante & -3.4 & 0.37 & 83.78 & 0 & 0.03 & & \\
Conducta violenta & 1.06 & 0.21 & 23.75 & 0 & 2.91 & 1.89 & 4.47 \\
& Constante & -2.58 & 0.34 & 55.05 & 0 & 0.07 & & \\
Implicación académica & Conducta violenta & 0.27 & 0.21 & 1.62 & 0.02 & 1.32 & 0.86 & 2.02 \\
Afiliación entre compañeros & Constante & -1.38 & 0.34 & 16.56 & 0 & 0.24 & & \\
& Conducta violenta & 0.98 & 0.21 & 21.69 & 0 & 2.68 & 1.77 & 4.07 \\
Apoyo del profesor & Constante & -1.98 & 0.33 & 36.08 & 0 & 0.13 & & \\
& Conducta violenta & 1.36 & 0.22 & 37.15 & 0 & 3.9 & 2.51 & 6.04 \\
& Constante & -2.94 & 0.35 & 68.64 & 0 & 0.05 & & \\
\hline
\end{tabular}

Fuente: elaboración propia

ajuste personal, se observan también incrementos en la probabilidad de presentar baja autoestima $(\mathrm{OR}=2.07)$, baja satisfacción vital $(\mathrm{OR}=2.86)$ y bajo grado de empatía $(\mathrm{OR}=5.85)$, conforme se incrementa la puntuación en conducta agresiva. Finalmente, los resultados referentes a las variables de ajuste escolar indican que por cada punto que aumenta la conducta agresiva, aumenta en 9.31 veces la probabilidad de que las estudiantes muestren una baja actitud positiva hacia la escuela y el profesorado, en 3.02 veces que presenten baja implicación académica, en 3.72 veces que informen de una baja afiliación con compañeros de clase y en 3.78 veces que perciban un bajo apoyo del profesor. En resumen y en el caso de las chicas, el orden de las variables según el valor de la OR de mayor a menor es el siguiente: baja actitud positiva hacia la escuela y el profesorado, bajo grado de empatía, bajo apoyo del profesor, baja afiliación entre compañeros, alta sintomatología depresiva, baja implicación académica, baja satisfacción vital, alto estrés percibido, alto sentimiento de soledad y baja autoestima.

\section{Discusión}

El presente estudio tuvo como objetivo determinar en qué medida la conducta agresiva hacia los iguales predice un mayor desajuste personal y escolar en los adolescentes agresores, en una muestra amplia de estudiantes españoles de ESO. La hipótesis planteada en este estudio postulaba que el comportamiento agresivo hacia los iguales actuaría como un predictor positivo y significativo de diferentes indicadores de ajuste personal y escolar, tanto en chicos como en chicas.

Los resultados confirmaron que, en ambos sexos, las puntuaciones en agresión hacia los iguales predicen significativamente las altas puntuaciones 
TABLA 2

Regresión logística con conducta violenta como predictor en chicas

\begin{tabular}{lcccccccc}
\hline Altas puntuaciones & & B & ET & Wald & p & OR & CI 95\% \\
\hline Sintomatología depresiva & & & & & & Inferior Superior \\
& Conducta violenta & 1.22 & 0.28 & 18.66 & 0 & 3.44 & 1.95 & 5.96 \\
Estrés percibido & Constante & -3.08 & 0.43 & 51.56 & 0 & 0.046 & & \\
& Conducta violenta & 1 & 0.3 & 1.56 & 0 & 2.73 & 1.48 & 4.98 \\
Sentimiento soledad & Constante & -3.21 & 0.47 & 46.61 & 0 & 0.04 & & \\
& Conducta violenta & 0.86 & 0.27 & 9.73 & 0 & 2.35 & 1.37 & 4.06 \\
& Constante & -2.44 & 0.41 & 34.98 & 0 & 0.087 & & \\
\hline Bajas puntuaciones & & & & & & & \\
\hline Autoestima general & Conducta violenta & 0.73 & 0.26 & 7.89 & 0 & 2.07 & 1.24 & 3.46 \\
& Constante & -1.82 & 0.38 & 22.47 & 0 & 0.16 & & \\
Satisfacción vital & Conducta violenta & 1.05 & 0.26 & 15.64 & 0 & 2.86 & 1.7 & 4.83 \\
& Constante & -2.33 & 0.39 & 34.94 & 0 & 0.09 & & \\
Grado de Empatía & Conducta violenta & 1.76 & 0.3 & 33.8 & 0 & 5.85 & 3.22 & 1.62 \\
& Constante & -4.09 & 0.46 & 76.44 & 0 & 0.02 & & \\
Actitud hacia escuela/profesorado & Conducta violenta & 2.23 & 0.3 & 53.56 & 0 & 9.31 & 5.12 & \multirow{2}{*}{16.92} \\
& Constante & -4.15 & 0.45 & 83.17 & 0 & 0.01 & & \\
Implicación académica & Conducta violenta & 1.10 & 0.27 & 16.83 & 0 & 3.02 & 1.78 & 5.12 \\
& Constante & -2.51 & 0.4 & 39.22 & 0 & 0.08 & & \\
Afiliación entre compañeros & Conducta violenta & 1.31 & 0.27 & 23.61 & 0 & 3.72 & 2.19 & 6.33 \\
& Constante & -2.45 & 0.39 & 38.18 & 0 & 0.08 & & \\
Apoyo del profesor & Conducta violenta & 1.33 & 0.27 & 23.35 & 0 & 3.78 & 2.2 & 6.48 \\
& Constante & -2.86 & 0.41 & 48.52 & 0 & 0.06 & & \\
\hline
\end{tabular}

Fuente: elaboración propia

en las dimensiones personales de sintomatología depresiva, estrés percibido y soledad y bajas puntuaciones en las dimensiones de autoestima, satisfacción con la vida y empatía. Además, también se encontró que la agresión predice bajas puntuaciones en las dimensiones escolares de actitud positiva hacia la escuela y el profesorado, implicación académica, afiliación con otros compañeros y apoyo percibido del profesor. Algunos de estos resultados son consistentes con los obtenidos en investigaciones previas, si bien añaden información en cuanto a indicadores específicos de ajuste personal y escolar que se asocian a la agresión entre iguales.

En relación con el desajuste personal, estudios previos también han encontrado que una mayor agresión hacia los iguales autoinformada se relaciona con más síntomas de ansiedad (Zimmer-Gembeck \& Pronk, 2012; Jiménez, Estévez, del Moral, \& Povedano, 2011) y depresión (Welch, 2008; Zimmer-Gembeck \& Pronk, 2012) y que si la agresión co-ocurre con depresión, predice la ideación suicida cuatro años después (Brunstein-Klomek et al., 2011). Además, los resultados del presente estudio sugieren que implicarse en conductas de agresión hacia los iguales puede disminuir progresivamente los niveles de satisfacción con la vida y empatía. En relación con esta última variable, según un estudio de Joliffe y Farrington (2006) ser un agresor frecuente (frente a ser agresor ocasional) se relaciona significativamente con baja empatía afectiva, tanto en hombres como en mujeres.

Otro resultado interesante es el relativo a la autoestima. En general, los adolescentes agresivos parecen tener una peor percepción de sí mismos (Inglés, Martínez-González, García-Fernández, Torregrosa, \& Ruiz-Esteban, 2012). Sin embargo, la relación entre autoestima y agresión y violencia resulta controvertida. Los resultados del presente estudio indican que la agresión predice la baja autoestima, si bien esta predicción es una de las que 
obtienen valores más bajos tanto en chicos como en chicas. En otros estudios se ha encontrado que los agresores tienen niveles altos de autoestima (Olweus, 1993), comparables a los no implicados en dinámicas de agresión hacia los iguales (Jiménez et al., 2011). Una posible explicación a estos resultados es que la relación entre autoestima y conducta violenta no es lineal y que son los valores extremos en autoestima (o muy bajos o muy altos) los que se relacionan con esta conducta (Ostrowsky, 2010). Algo semejante podría ocurrir con la soledad, ya que si bien se ha observado una asociación significativa con la agresión, obtiene valores bajos de predicción. En otros estudios se ha constatado que los adolescentes agresores informan de un menor sentimiento de soledad que las víctimas (Jiménez et al., 2011), por lo que podría considerarse que la agresión, a corto plazo, parece ofrecer ciertos beneficios en la percepción de cercanía y conexión con un cierto grupo de iguales.

En relación con el desajuste escolar, por un lado, destacan la baja actitud positiva hacia la institución escolar y el profesorado y el bajo apoyo percibido de este. Estos resultados son coherentes con estudios previos (Vanderbilt \& Augustyn, 2010) y con la idea de que los adolescentes que se implican en conductas de agresión en la escuela no confían en la institución y podrían implicarse en este tipo de comportamientos como una medida de autoprotección cuando se sienten inseguros (Estévez, Jiménez, Moreno, \& Musitu, 2013). En este caso, frente a la punición institucional por su conducta, podrían desarrollar actitudes aún más negativas hacia la escuela y el profesorado. Por otro lado, la percepción del clima del aula en términos de sentimiento de afiliación y amistad entre compañeros e implicación en las actividades escolares también se ve afectada por las conductas de agresión entre iguales. En este sentido, Letamendía (2002) señala que la presencia de poder y asimetría en las relaciones entre escolares provoca un daño para todos los implicados; agresores, víctimas y observadores, porque mina la horizontalidad en las interacciones entre iguales cuya experiencia durante los años escolares constituye una tarea evolutiva necesaria para un adecuado desarrollo personal.
Los resultados también revelan algunas diferencias en función del sexo, como por ejemplo que, por orden de importancia, la agresión sea predictiva de la baja actitud hacia la autoridad institucional y el profesorado en primer lugar para las chicas y solo en sexto lugar para los chicos. Es posible que este tipo de resultados de diferencias en función del sexo pueda explicarse en relación con la hipótesis de la paradoja del género (Keenan, Loeber, \& Green, 1999), según la cual los adolescentes que desarrollan de forma intensa desórdenes atípicos de su género (por ejemplo, la agresión en las chicas) presentan conductas mucho más desviadas que aquellos adolescentes que desarrollan desordenes típicos de su género (por ejemplo, la agresión en los chicos). Aplicando esta hipótesis a los resultados presentes, se podría sugerir que las chicas con mayores niveles de comportamientos de agresión hacia sus iguales desarrollan unas actitudes mucho más negativas hacia la autoridad institucional y el profesorado que los chicos que tienen niveles semejantes de agresión hacia los iguales. Sin embargo, las diferencias de sexo en la importancia de algunas variables en relación con la agresión (actitud hacia la autoridad, sintomatología depresiva, estrés percibido) apenas ha sido analizada en la literatura científica y es necesaria una mayor investigación para poder ofrecer explicaciones más consistentes de estas diferencias entre sexos.

En resumen, este estudio ha mostrado que, en estudiantes de ESO, tanto varones como mujeres, la conducta de agresión hacia los iguales está relacionada con distintos indicadores de desajuste personal y escolar. Estos resultados apoyan la idea de que es importante que la urgencia de tratar a las víctimas (reparación del daño) no soslaye la importancia de trabajar con los agresores, ya que esta conducta problemática tiene un mal pronóstico, no solo porque la agresión hacia los iguales en la escuela incrementa en dos tercios la probabilidad de tener otras conductas violentas pasados algunos años (Ttofi et al., 2012), sino porque también incrementa la probabilidad de abandono escolar y de ser diagnosticados con desordenes psiquiátricos de conducta antisocial, abuso de sustancias y cuadros ansiosos (Vanderbilt \& Augustyn, 2010). 
Los resultados ponen en evidencia que la intervención con los agresores debe ser integral, contemplando tanto el desajuste personal (autovaloración, sintomatología depresiva y ansiosa, etc.) como el escolar del estudiante (integración en el aula, relación con el profesor, actitudes hacia la institución, etc.). En el caso de que se contemple una intervención meramente punitiva (por ejemplo, la expulsión del centro), es muy probable que se produzca un efecto de retroalimentación en el que el estudiante se sienta cada vez más alejado de la institución escolar, de sus integrantes y del proceso de enseñanza-aprendizaje, con la subsiguiente pérdida de oportunidades para su adecuado desarrollo psicosocial.

El presente estudio también tiene limitaciones que futuros trabajos deberían intentar subsanar. En primer lugar, los resultados obtenidos no pueden generalizarse a estudiantes de otros niveles educativos como Educación Primaria, en los que sabemos que también se observan problemas de agresión escolar. En segundo lugar, es importante resaltar que en esta investigación se han empleado únicamente medidas de autoinforme, lo cual puede introducir sesgos derivados de la deseabilidad social, aspecto este particularmente importante cuando se evalúa la agresión, ya que los estudiantes podrían minimizar o, al contrario, maximizar este tipo de comportamiento. En este sentido, la investigación futura podría utilizar otros procedimientos de evaluación como escalas de valoración de la agresión por los iguales que permitirían contrastar la información. Finalmente, la naturaleza transversal del presente estudio impide el establecimiento de inferencias causales respecto a las relaciones entre la agresión hacia los iguales y los indicadores de ajuste personal y escolar. Sería aconsejable utilizar diseños longitudinales para confirmar la dirección de las relaciones.

\section{Referencias}

Álvarez-García, D., Álvarez, L., Núñez, J. C., González, P., González-Pienda, J. A., Rodríguez, C., \& Cerezo, R. (2010). Violencia en los centros educativos y fracaso académico. Revista Iberoamericana de Psicología y Salud, 1(2), 139-153.
Astor, R., Pitner, R. O., Benbenishty, R., \& Meyer, H. A. (2002). Public concern and focus on school violence. En L. A. Rapp-Paglicci, A. R. Roberts \& J. S. Wodarski (Eds.), Handbook of violence (pp. 262-302). New York: Wiley.

Atienza, F. L., Pons, D., Balaguer, I., \& García-Merita, M. (2000). Propiedades psicométricas de la escala de satisfacción con la vida en adolescentes. Psicothema, 12(2), 314-320.

Blaya, C., Debardieux, E., del Rey, R., \& Ortega, R. (2006). Clima y violencia escolar. Un estudio comparativo entre España y Francia. Revista de Educación, 339, 293-315.

Brunstein-Klomek, A., Kleinman, M., Altschuler, E., Marocco, F., Amakawa, L., \& Gould, M. S. (2011). High school bullying as a risk for later depression and suicidality. Suicide and Life-Threatening Behavior, 41(5), 501-516.

Bryant, B. K. (1982). An index of empathy for children and adolescents. Child Development, 53(2), 413. 425.

Cava, M. J., Musitu, G., \& Murgui, S. (2007). Individual and social risk factors related to overt victimization in a sample of Spanish adolescents. Psychological Reports, 101(1), 275-290.

Cohen, S., Kamarck, T., \& Mermelstein, R. (1983). A global measure of perceived stress. Journal of Health and Social Behavior, 24(4), 385-396.

De Maris, A. (2003). Logistic regression. En J. A. Schinka \& W. F. Velicer (Eds.), Research methods in psychology (pp. 509-532). New Jersey: John Wiley y Sons.

Diener, E., Emmons, R., Larsen, R. J., \& Griffin, S. (1985). The Satisfaction With Life Scale. Journal of Personality Assessment, 49(1), 71-75.

Echeburúa, E. (1995). Evaluación y tratamiento de la fobia social. Barcelona: Martínez Roca.

Emler, N. (2009). Delinquents as a minority group: Accidental tourists in forbidden territory or voluntary émigrés? En F. Butera \& J. Levine (Eds.), Coping with minority status: Responses to exclusion and inclusion (pp. 127-154). Cambridge: Cambridge University Press.

Esteban-Guitart, M., Rivas, M. J., \& Pérez, M. R. (2011) Empatía y tolerancia a la diversidad en un contexto 
educativo intercultural. Universitas Psychologica, 11(2), 415-426.

Estévez, E., Herrero, J., Martínez, B., \& Musitu, G. (2006). Aggressive and non-aggressive rejected students: An analysis of their differences. Psychology in the Schools, 43(3), 387-400.

Estévez, E., Inglés, C. J., \& Martínez-Monteagudo, M. C. (2013). School aggression: Effects of classroom environment, attitude to authority and social reputation among peers. European Journal of Investigation in Health, Psychology and Education, 3(1), 22-32.

Estévez, E., Jiménez, T., \& Musitu, G. (2008). Violence and victimization at school in adolescence. En D. H. Molina (Ed.), School psychology: 21st century issues and challenges (pp. 79-115). New York: Nova Science Publishers.

Estévez, E., Moreno, D., Jiménez, T., \& Musitu, G. (2013). From victim to aggressor: An analysis of the relationship between victimization and violent behavior at school. Spanish Journal of Psychology, 16(1), 1-13.

Estévez, E., Murgui, S., \& Musitu, G. (2008). Psychosocial adjustment in aggressors, pure victims and aggressive victims at school. European Journal of Education and Psychology, 1(2), 29-39.

Evans, M., Heriot, S. A., \& Friedman, A. G. (2002). A behavioural pattern of irritability, hostility and inhibited empathy in children. Clinical Child Psychology and Psychiatry, 7(2), 211-224.

Expósito, F., \& Moya, M. (1993). Validación de la UCLA Loneliness Scale en una muestra española. En F. Loscertales \& M. Marín (Eds.), Dimensiones psicosociales de la educación y de la comunicación (pp. 355-364). Sevilla: Eudema.

Fernández-Ballesteros, R., \& Sierra, B. (1989). Escalas de Clima Social FES, WES, CIES y CES. Madrid: TEA.

Gázquez, J. J., \& Pérez-Fuentes, M. C. (2010). Violencia escolar y rendimiento académico: instrumento para diagnosticar el bajo rendimiento asociado a los perfiles de violencia en los centros. En J. J. Gázquez \& M. C. Pérez-Fuentes (Coords.), La convivencia escolar. Aspectos psicológicos y educativos (pp. 333 338). Granada: GEU.

Herrero, J., \& Meneses, J. (2006). Short web-based versions of the Perceived Stress (PSS) and Center for Epidemiological Studies-Depression (CESD) Scales: A comparison to pencil and paper responses among Internet users. Computers in Human Behavior, 22, 830-848.

Hunter, S. C., Mora-Merchan, J., \& Ortega, R. (2004). The long-term effects of coping strategy use in victims of bullying. The Spanish Journal of Psycho$\log y, 7(1), 3-12$.

Inglés, C. J., Martínez-González, A. E., García-Fernández, J. M., Torregrosa, M. S., \& Ruiz-Esteban, C. (2012). Prosocial behavior and self-concept of Spanish students of compulsory secondary education. Revista de Psicodidáctica, 17(1), 135-156.

Jiménez, T. I., Estévez, E., del Moral, G., \& Povedano, A. (2011). Violencia y victimización entre iguales: factores de riesgo y protección en la familia, la escuela y la comunidad. En R. Pereira (Comp.), Adolescentes en el siglo XXI: entre impotencia, resiliencia y poder (pp. 403-425). Madrid: Morata.

Jolliffe, D., \& Farrington, D. P. (2006). Examining the relationship between low empathy and bullying. Aggressive Behavior, 32(6), 540-550.

Juvonen, J., Nishina, A., \& Graham, S. (2000). Peer harassment, psychological adjustment, and school functioning in early adolescence. Journal of Educational Psychology, 92(2), 349-359.

Keenan, K., Loeber, R., \& Green, S. (1999). Conduct disorder in girls: A review of the literature. Clinical Child and Family Psychology Review, 2(1), 3-19.

Letamendía, R. (2002). El maltrato en contextos escolares. Revista de Psicodidáctica, 13, 77-90.

Little, T. D., Henrich, C. C., Jones, S. M., \& Hawley, P. H. (2003). Disentangling the "whys" from the "whats" of aggressive behaviour. International Journal of Behavioral Development, 27(2), 122-131.

Martínez, B., Murgui, S., Musitu, G., \& Monreal, M. C. (2009). El rol del apoyo parental, las actitudes hacia la escuela y la autoestima en la violencia escolar en adolescente. International Journal of Clinical and Health Psychology, 8(3), 679-692.

Martínez, B., Povedano, A., Amador, L. V., \& Moreno, D. (2012). Clima escolar, satisfacción con la vida y victimización en la escuela. Un análisis del efecto moderador del género. Anales de Psicología, 28(3), 875-882. 
Mestre, V., Pérez-Delgado, E., Frías, D., \& Samper, P. (1999). Instrumentos de evaluación de la empatía. En E. Pérez-Delgado \& V. Mestre (Coords.), Psicología moral y crecimiento personal (pp.181-190). Barcelona: Ariel.

Mestre, V., Samper, P., Tur-Porcar, A. M., Richaud de Minci, M. C., \& Mesurado, B. (2012). Emociones, estilos de afrontamiento y agresividad en la adolescencia. Universitas Psicologica, 11(4), 1263-1275.

Molpeceres, M. A., Lucas, A., \& Pons, D. (2000). Experiencia escolar y orientación hacia la autoridad institucional en la adolescencia. Revista de Psicología Social, 15(2), 87-105.

Moos, R. H., \& Trickett, E. J. (1973). Classroom Environment Scale manual. Palo Alto, CA: Consulting Psychologist Press.

Moreno, D., Estévez, E., Murgui, S., \& Musitu, G. (2009). Relación entre el clima familiar y el clima escolar: el rol de la empatía, la actitud hacia la autoridad y la conducta violenta en la adolescencia. International Journal of Psychology and Psychological Therapy, 9(1), 123-136.

Murray, C., \& Murray, K. M. (2004). Child level correlations of teacher-students relationships: An examination of demographic orientation characteristics, academia orientations and behavioral orientations. Psychology in the Schools, 41(7), 751-762.

Nansel, T. R., Overpeck, M., Pilla, R. S., Ruan, W. J., Simons-Morton, B., \& Scheidt, P. (2001). Bullying behaviors among US youth: Prevalence and association with psychosocial adjustment. Journal of the American Medical Association, 285(16), 2094-2100.

Olweus, D. (1993). Bullying at school: What we know and what we can do. Oxford: Blackwell.

Olweus, D. (2005). Bullying en la escuela: datos e intervención. En J. Sanmartín (Ed.), Violencia y escuela (pp. 13-30). Valencia: Centro Reina Sofía para el Estudio de la Violencia.

Olweus, D. (2011). Bullying at school and later criminality: Findings from three Swedish community samples of males. Criminal Behaviour and Mental Health, 21(2), 151-156.

Ostrowsky, M. K. (2010). Are violent people more likely to have low self-esteem or high self-esteem? Aggression and Violent Behavior, 15(1), 69-75.
Postigo, S., González, R., Mateu, C., Ferrero, J., \& Martorell, C. (2009). Diferencias conductuales según género en convivencia escolar. Psicothema, 21(3), 453-458.

Povedano, A., Estévez, E., Martínez, B., \& Monreal, M. C. (2012). Un perfil psicosocial de adolescentes agresores y víctimas en la escuela: análisis de las diferencias de género. Revista de Psicología Social, 27(2), 169-182.

Radloff, L. S. (1977). The CES-D Scale: A self-report depression scale for research in the general population. Applied Psychological Measurement, 1, 385- 401.

Reicher, S., \& Emler, N. (1985). Delinquent behaviour and attitudes to formal authority. British Journal of Social Psychology, 3, 161-168.

Reinke, W. M., \& Herman, K. C. (2002). Creating school environment that deter antisocial behaviors in youth. Psychology in the Schools, 39(5), 549-559.

Rosenberg, M. (1965). Society and the adolescent selfimage. Princeton, NJ: Princeton University Press.

Russell, D., Peplau, L. A., \& Cutrona, C. E. (1980). The revised UCLA Loneliness Scale: Concurrent and discriminant validity evidence. Journal of Personality and Social Psychology, 39(3), 472-480.

Sweeting, H., Young, R., West, P., \& Der, G. (2006). Peer victimization and depression in early-mid adolescence: A longitudinal study. British Journal of Educational Psychology, 76(3), 577-594.

Ttofi, M. M., Farrington, D. P., \& Lösel, F. (2012). School bullying as a predictor of violence later in life: A systematic review and meta-analysis of prospective longitudinal studies. Aggression and Violent Behavior, 17(5), 405-418.

Vanderbilt, D., \& Augustyn, M. (2010). The effects of bullying. Paediatrics and Child Health, 20(7), 315-320.

Villarreal-González, M. E., Sánchez-Sosa, J. C., Veiga, F. H., \& del Moral Arroyo, G. (2011). Development contexts, psychological distress, social self-esteem and school violence from a gender perspective in Mexican adolescents. Psychosocial Intervention, 20(2), 171-181.

Welch, T. R. (2008). Bullies and their victims. The Journal of Pediatrics, 152 (1), A1. 
Wild, L. G., Flisher, A. J., Bhana, A., \& Lombard, C. (2004). Associations among adolescent risk behaviours and self-esteem in six domains. Journal of Child Psychology and Psychiatry, 45(8), 1454-1467.
Zimmer-Gembeck, M. J., \& Pronk, R. E. (2012). Relation of depression and anxiety to self- and peerreported relational aggression. Aggressive Behavior, 38(1), 16-30. 
